\title{
Laparoscopic splenic cystectomy
}

\author{
Madushanka UDP, Jayathilaka A, Herath K, Galketiya KB \\ University Surgical Unit, Teaching Hospital Peradeniya, Sri Lanka.
}

Correspondence: $\quad$ Dr. U.D.P. Madushanka

e-mail: piyummadushanka@gmail.com

(D) https://orcid.org/0000-0003-4941-854X

\section{Introduction}

Splenic cyst is a rare condition identified and characterised by imaging methods such as ultrasound and computerised tomography in patients presenting with upper abdominal discomfort. The classification of splenic cysts is based on the work of Fowler and Martin (1). Splenic cysts classified as Type I primary or "true" cysts, or as Type II secondary splenic cysts or "pseudocysts."

Splenic cysts larger than $5 \mathrm{~cm}$ are symptomatic and need excision for diagnostic purposes. Laparoscopic excision preserving spleen is preferred as it is associated with lower morbidity. We present a case report of a twenty six year old female who underwent a laparoscopic excision for a splenic cyst.

\section{Case report}

A 26-year old female presented with vague upper abdominal pain and distention. On cross contrast enhanced sectional imaging she was found to have a large splenic cyst (Figure 1).

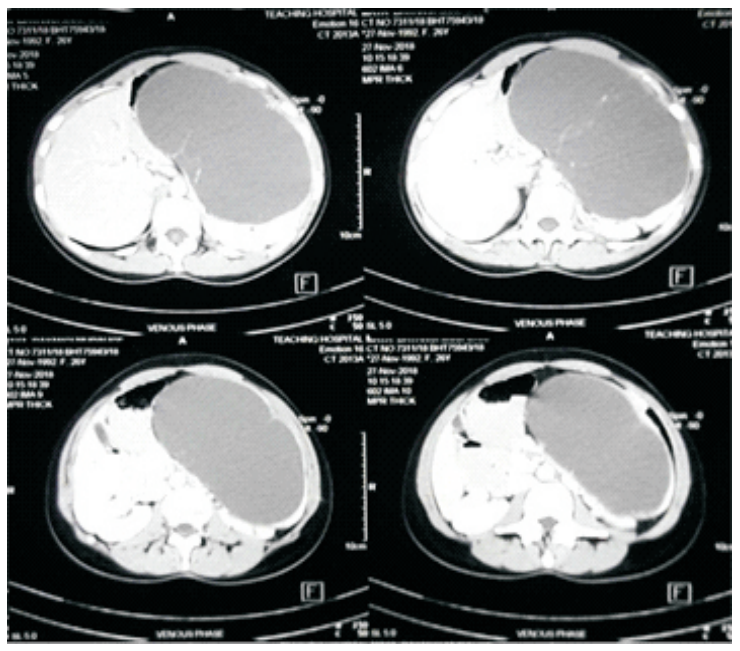

Figure 1: Cross sectional CT imaging of the cyst
Cyst was subjected to ultrasound guided aspiration and the cytological analysis of aspirated fluid showed benign cells. Repeat ultrasound scan in 2 months revealed reappearing of the cyst. Informed consent was obtained for laparoscopic excision.

\section{Procedure \\ Patient Positioning}

After general anesthesia with endotracheal intubation patient was placed on right lateral position with flexion of right upper limb over the chest to expose the left side of the abdomen.

\section{Ports Placement}

Three $5 \mathrm{~mm}$ ports and two $10 \mathrm{~mm}$ ports were inserted using following landmarks (Figure 2).

\section{Identifying and demonstration of anatomical landmarks}

Following anatomical structures were identified; stomach, colon, spleen with cyst, gastrocolic and gastrosplenic ligaments.

\section{Surgical Dissection}

Cyst contents were aspirated using a sucker. Nearly $900 \mathrm{ml}$ of straw coloured fluid was aspirated. The aspiration facilitated dissection. Gastrocolic and gastrosplenic ligaments were divided by ultrasonic dissector. Cyst wall was identified separately from splenic tissue and dissected by using an ultrasonic dissector and bipolar diathermy. In some areas a thin slice of splenic tissue had to be incorporated to ensure complete excision. Specimen was then retrieved through $10 \mathrm{~mm}$ port after small extension 
of the incision. A tube drain was inserted through $5 \mathrm{~mm}$ port.

The patient was managed on the first day in the high dependency unit. She had minimal analgesic requirements and oral feeding was commenced on same day. She was discharged on the fourth postoperative day.

Histopathology revealed a benign primary epithelial cyst with a cuboidal cell lining no aetiological factor revealed.

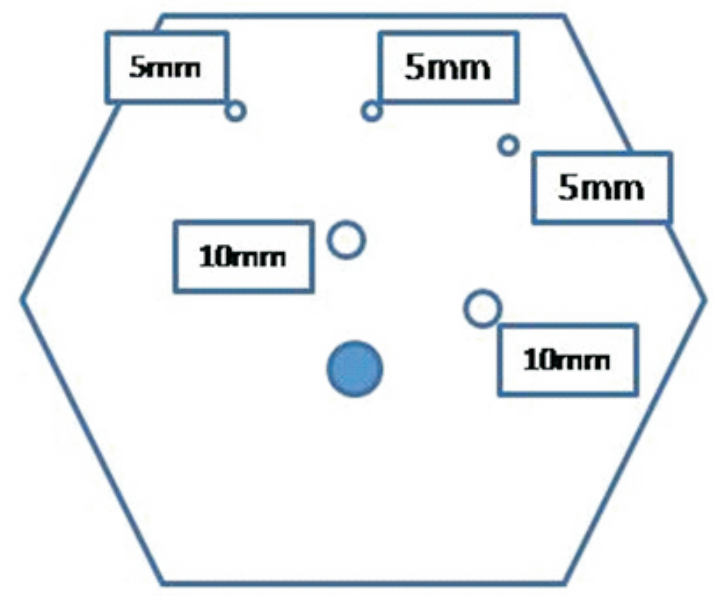

Figure 2: Port positioning for the procedure

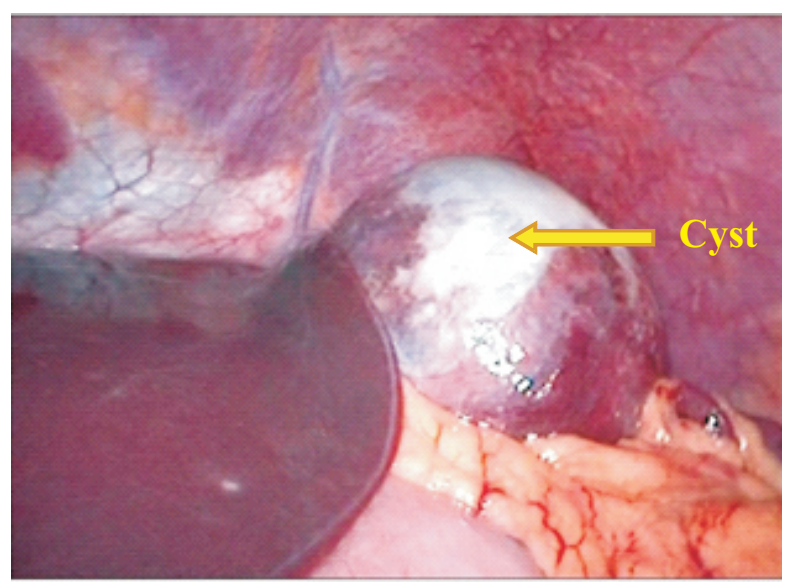

Figure 3: Laparoscopic appearance of the splenic cyst

\section{Discussion}

The occurrence of splenic cysts is rare. Most are asymptomatic and detected incidentally.

Splenic cysts are classified as True cysts (Type I) and Pseudocysts (Type II). Type I cysts are distinguished from Type II cysts by the presence of an epithelial lining. Primary or true cysts include both parasitic and nonparasitic aetiologies. Nonparasitic cysts can be further subdivided into congenital (epithelial), vascular, and neoplastic cysts. Type II "pseudocysts" are most commonly due to blunt trauma, infection or infarction.

According to size and symptoms, most of them can be managed conservatively with serial imaging at follow up. But large symptomatic cysts need surgical management which is diagnostic and therapeutic. Indications for operative intervention include cysts with a diameter $>5 \mathrm{~cm}$ and those which are symptomatic (2). Open surgery was the mainstay of treatment in the past and spleneccystectomy was considered as the treatment of choice. With the introduction of minimally invasive surgery laparoscopic methods have gradually evolved (3). Salky et al., first reported laparoscopic deroofing of a splenic cyst in 1985 (4). It has reduced morbidity when compared with the traditional laparotomy approach (5). The patient being discussed here had a large symptomatic cyst which was successfully excised, laparoscopically, conserving the spleen.

\section{Conclusions}

Laparoscopic removal of benign splenic cysts offers an effective and minimally invasive alternative to the traditional laparotomy. It allows reduction in postoperative pain and length of hospitalization, improved cosmesis, minimal bleeding and preservation of splenic function.

\section{References}

1. Martin JW. Congenital splenic cysts. Am J Surg, 1958; 96: 302-8. [PubMed] [Google Scholar]

2. Katherine A Barsness, Marleta Reynolds. The Spleen. In: Pediatric Surgery ( $7^{\text {th }}$ Edition), 2012; Chap 110; 1385-1392.

3. Gianom D, Wildisen A, Hotz T, Goti F, Decurtins M. Open and Laparoscopic Treatment of Nonparasitic Splenic Cysts 2003; 20(1): (DOI:10.1159/000068860)

4. Salky B, Zimmerman M, Bauer J, Gelernt I, Kreel I. Splenic cyst: Definitive treatment by laparoscopy. Gastrintest Endosc, 1985; 31:213. [PubMed] [Google Scholar]

5 Jeffrey B. Comitalo. Laparoscopic Treatment of Splenic Cysts. JSLS, 2001; Oct-Dec; 5(4): 313-316. 